\begin{tabular}{|l|l|c|c|}
\hline Eiszeitalter u.Gegenwart & 30 & $\begin{array}{c}229-236 \\
3 \mathrm{Abb} .\end{array}$ & Hannover 1980 \\
\hline
\end{tabular}

\title{
Meeresspiegeldaten aus \\ landschafts- und siedlungsgeschichtlichen Untersuchungen auf Pellworm (Nordfriesische Inseln)
}

\author{
Dietrich HofFMANN *)
}

Sea level, landscape history, settlement history, C-14-dating, dendrochronologie, Holocene

North-West German Lowlands (Pellworm island), Schleswig Holstein. TK 25 Nr.: 1417

$\mathrm{Kurzf}$ assung: Es wird über archäologische, geographische und geologische Arbeiten zur Landschafts- und Besiedlungsgeschichte des südlichen nordfriesischen Wattenmeeres berichtet. Bei der Untersuchung von mittelalterlichen und frühneuzeitlichen Siedlungsplätzen und deren Untergrund auf der Insel Pellworm konnten u. a. neue Daten zur örtlichen Veränderung des Meeresspiegels im Holozän nachgewiesen werden: Danach hatte das Mittlere Tidehochwasser bereits in der zweiten Hälfte des letzten Jahrtausends vor Chr. Geb. eine Höhe von NN + 0,8 m erreicht, fiel danach jedoch wieder $a b$ und stieg mit Beginn des zweiten Jahrtausends nach Chr. Geb. wieder an.

\section{[Sea-Level-Datas Resulting from Investigations on the History of Landscape and Settlement on the Isle of Pellworm (North-Frisian Islands)]}

A b s r a c t : Since 1975 investigations on the history of landscape and settlement have been carried out in the southern North-Frisian tidal flats by an archeological, geographical and geological team. Excavations of medieval and early modern dwelling-mounds on the Isle of Pellworm proved new datas of local holocene sea-level changes: The level of mean high water had risen up to $\mathrm{NN}+0,8 \mathrm{~m}$ in the second half of the last millennium BC. In the beginning of the following millennium it was lower and began to rise again in the first centuries of the second millennium AD.

\section{Allgemeiner Überblick}

An der Westküste Schleswig-Holsteins werden im Bereich der südlichen Nordfriesischen Inseln seit 1975 Untersuchungen zur Landschafts- und Siedlungsgeschichte durchgeführt (Higelke, Hoffmann \& Müller-Wille 1976; Higelke, Hoffmann, KüHN \& Müller-Wille 1979). Das Interesse richtet sich auf ein Gebiet, das in den letzten 600 Jahren Veränderungen seiner Umwelt erfahren hat wie kaum ein anderes. Dies ist vor allem auf Sturmfluten im 14. und 17. Jahrhundert zurückzuführen, die in große, seit langer Zeit eingedeichte, tief liegende Wirtschafts- und Siedlungsflächen an so vielen Stellen einbrachen, daß eine erneute Sicherung aller dieser Gebiete nicht mehr möglich war und der größte Teil daher bis heute im Einflußbereich der Tiden verblieb. So sind die heutigen Inseln Nordstrand und Pellworm sowie die Hallig Nordstrandischmoor Reste einer ursprünglich mehr oder weniger eng mit dem Festland zusammenhängenden Marsch gewesen, die im 14. Jahrhundert durch Sturmfluten zerrissen wurden, wobei die Insel AltNordstrand, in der die drei oben genannten Inseln noch vereinigt waren, entstand. Im 17. Jahrhundert wurde auch diese Insel durch Sturmfluten zerstückelt, bei denen bereits Wasserstände wie bei der Sturmflut 1962 (Prange 1965) erreicht wurden (BANTElmanN 1966). Zeugen dieses Geschehens sind die heute noch im Watt sichtbaren Spuren der Kultivierungsarbeiten und Siedlungsplätze.

*) Anschrift des Verfassers: Dr. D. Hof $\mathrm{f} m$ a $\mathrm{n} n$, Marschen- und Wurtenforschung von Schleswig-Holstein, Schloß Gottorp, D-2380 Schleswig. 
Bei der Suche nach den Ursachen der hier aufgezeigten Entwicklung werden folgende Aspekte diskutiert:

1. Der säkulare Anstieg des Mittleren Tidehochwassers sowie der Anstieg der Sturmfluthöhen.

2. Der Eingriff des Menschen in den Bodenaufbau durch Kultivierungsmaßnahmen und Rohstoffgewinnung (Torf).

3. Der Eingriff des Menschen in die hydrographischen Verhältnisse durch umfangreiche Eindeichungen.

4. Wirkung der Eindeichungen auf das Sedimentationsgeschehen, wie z. B. Verhinderung des Aufwachsens der Oberflächen.

Aus der Erkenntnis heraus, daß zu diesen Fragen nur Lösungen in fachübergreifender Zusammenarbeit gefunden werden können, wurden drei Arbeitsgruppen, eine archäologische, eine geographische und eine geologische gebildet, die sich folgende Aufgaben stellten:

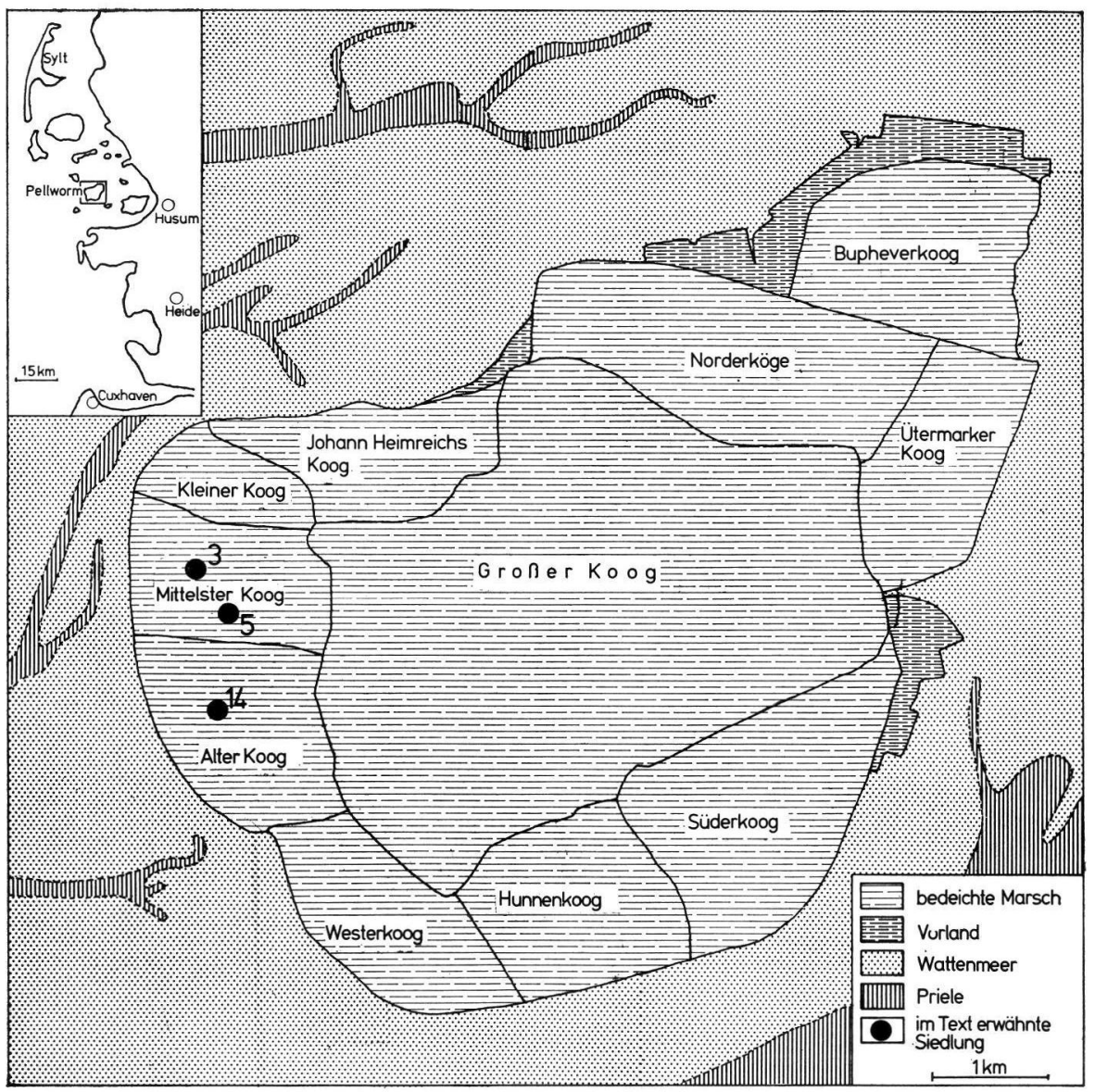

Abb. 1: Lageplan der untersuchten Siedlungen auf Pellworm. 
Ziel der geographischen Untersuchungen ist die Aufnahme mittelalterlich-frühneuzeitlicher Kulturlandschaftsreste (Kartierung, Befliegung, Auswertung historischen und modernen Kartenmaterials) in den Wattgebieten, die Rekonstruktion verschiedener Phasen der Landschaftsentwicklung sowie die Analyse und Darstellung der morphodynamischen Verhältnisse im Bereich des Wattenmeeres, der Priele und der Wattströme. Dazu stehen insgesamt drei zeitliche Stadien nivellitischer Aufnahmen der Wattengebiete zur Verfügung, die mit Unterstützung des Rechenzentrums an der Universität Regensburg für die Untersuchungen der rezenten Morphodynamik ausgewertet werden sollen, darüber hinaus werden auch die auf Luftbildern verschiedenen Alters sichtbaren morphologischen Details ausgewertet.

Ziel der siedlungsarchäologischen Untersuchungen ist die Erhellung des eng mit der Landschaftsentwicklung verknüpften Besiedlungsbeginns und -verlaufs. Dazu sind bisher 11 Warften und Flachsiedlungen auf Pellworm, Nordstrand, Langeneß und Hooge durch Schnittgrabungen untersucht worden. Diesen Arbeiten ging eine Aufnahme aller feststellbaren Siedlungsstellen und sonstigen obertägigen Bodendenkmäler voraus. Die bisherigen Ergebnisse der Arbeiten lassen eine Besiedlung seit dem 9./10. Jahrhundert, weiterhin die Anlage von Flachsiedlungen und Warften im hohen und späten Mittelalter sowie in der frühen Neuzeit erkennen, auf Pellworm in ungeregelter, auf Nordstrand teilweise in Form der Marschhufensiedlung.

Ziel der geologischen Arbeiten ist es, mit den der Geologie eigenen Arbeitsmethoden an der Rekonstruktion der Landschaftsentwicklung mitzuarbeiten und diese in Zeiten zu verfolgen, die in diesem Gebiet der archäologischen Forschung nicht mehr zugänglich sind. Für die jüngeren Zeiträume erfolgt dies in enger Kooperation mit den beiden anderen Arbeitsgruppen. Dabei soll vor allem Ausmaß, Art und Zweck der menschlichen Eingriffe in die Landschaftsentwicklung geklärt werden, um Alter und Höhenlage kultivierter Oberflächen im Hinblick auf Meeresspiegelstände des entsprechenden Zeitraums richtig beurteilen zu können. Außerdem sind aus der Untersuchung der gesamten zwischen $20 \mathrm{~m}$ und $7 \mathrm{~m}$ mächtigen Schichtfolge des Küstenholozäns Aussagen zum örtlichen Ablauf der nacheiszeitlichen Meerestransgression möglich. Diese waren vor allem aus der Untersuchung des Untergrundes der Siedlungsplätze zu erwarten, da unter den älteren noch Schichtprofile vorhanden sein mußten, die nicht durch Kultivierungsmaßnahmen gestört waren (Higelke, Hoffmann, KüHn \& Müller-Wille 1979). Außerdem gelang es, durch Höhenvergleiche Sackungs- und Setzungsbeträge abzuschätzen und so weitgehend die ursprüngliche Höhenlage der betrachteten Sedimentoberflächen anzugeben. Dazu eigneten sich besonders die gemeinsam mit den Archäologen vorgenommenen Grabungen an den Siedlungsplätzen 3, 5 und 14 im Westen der Insel Pellworm.

\section{Darstellung der Befunde}

Für die Untersuchungen waren quer durch die Wohnplätze mit einem Bagger Schnittgräben gelegt worden, deren Sohle bei etwa NN -1 m lag, so daß auch noch die anstehenden Schichten teilweise bis zu einer Mächtigkeit von 1,5 m aufgeschlossen wurden.

Die älteste untersuchte Stelle war eine Flachsiedlung aus dem 9.-10. Jahrhundert (Abb. 1, Punkt 3), die auf einer natürlichen, etwa $30 \mathrm{~cm}$ über die Umgebung herausragenden Kuppe angelegt war. Die Unterkante der Kulturschicht liegt an den höchsten Punkten bei NN $+0,6 \mathrm{~m}$ und fällt nach den Seiten bis auf $\mathrm{NN}+0,1 \mathrm{~m}$ ab (Abb. 2). Sie liegt auf einem 3-4 cm mächtigen Bodenhorizont, der nur noch unter der Kulturschicht erhalten ist; er bedeckt eine $50-60 \mathrm{~cm}$ mächtige Lage aus Sturmflutschichten. Darunter folgt ein 1,2 m mächtiger toniger, z. T. schluffig-feinsandiger Klei, der im unteren Teil durch Bioturbation völlig entschichtet ist. Die erwähnte Kulturschicht des 9./10. Jahrhunderts 


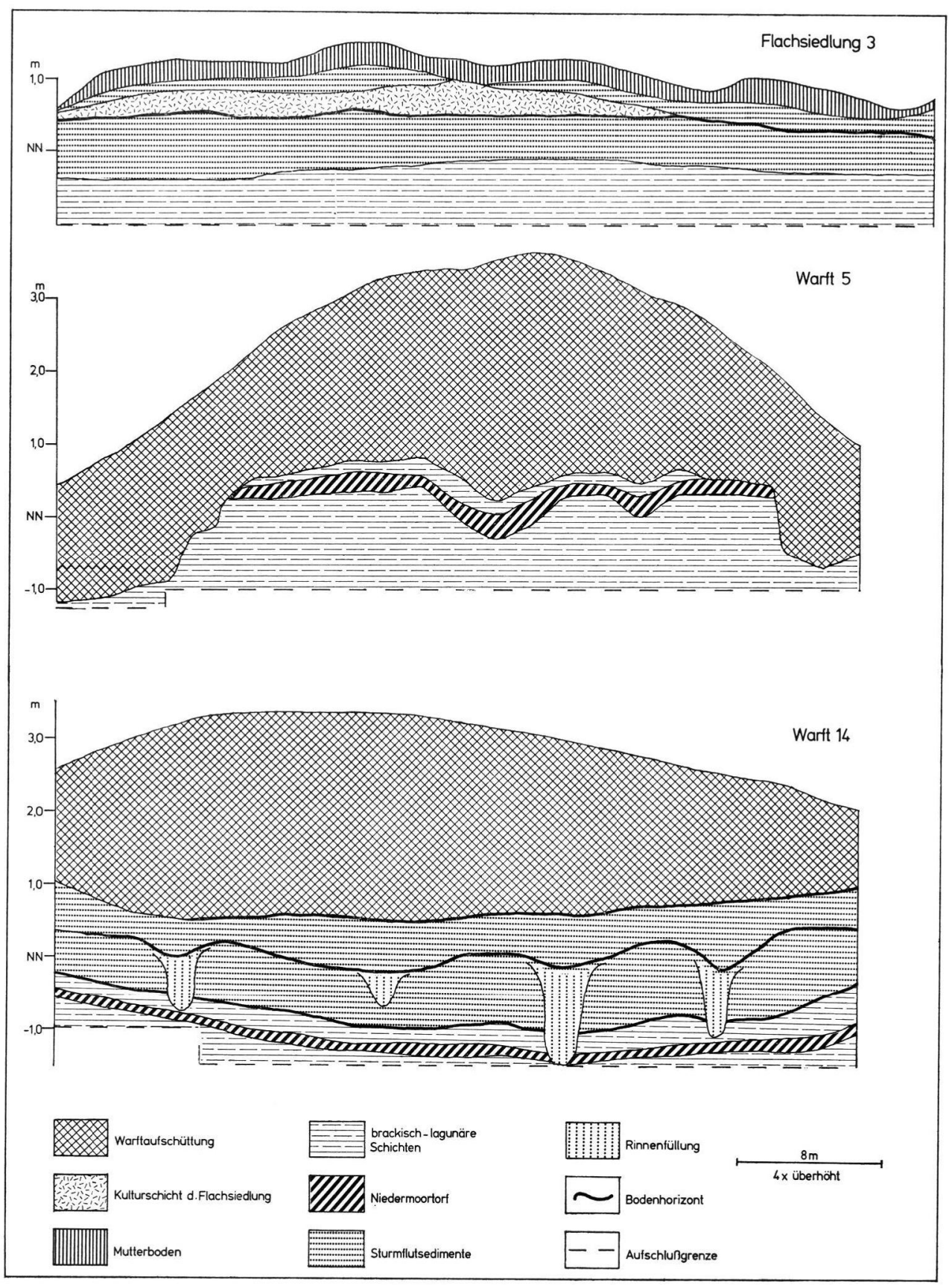

Abb. 2: Untersuchte Profile, nach der Geländedokumentation von H. J. KüHN (Siedlungen 3 und 5) und K. H. Dittmann (Siedlung 14) generalisierte Umzeichnung. 
wird ebenfalls von Sturmflutschichten überlagert, die an ihrer höchsten Stelle NN $+1,0 \mathrm{~m}$ erreichen. Sie enthalten Scherben des 12./13. Jahrhunderts. Darüber folgen ungefähr $20 \mathrm{~cm}$ Mutterboden.

War der Punkt 3 bei der Aufnahme der Bodendenkmäler durch seine flache, große Kuppe aufgefallen (Abb. 1), so erregte am Punkt 5 eine ungewöhnlich hohe Warft die Aufmerksamkeit. Bohrungen im Rahmen der Voruntersuchungen der Warften hatten gezeigt, daß der Warftkörper teilweise aus Torf besteht und auch selbst auf anstehenden Torf aufgesetzt ist (Abb.2). Die nähere Untersuchung durch den Baggerschnitt ergab folgende Befunde: Über einem stark mit Schilf durchsetzten, tonigen Klei liegt eine 20 bis $40 \mathrm{~cm}$ mächtige Niedermoorschicht, die im oberen Teil auch Holzstücke enthält. Ihre Oberkante liegt zwischen $\mathrm{NN}$ und $\mathrm{NN}+0,6 \mathrm{~m}$. Darüber folgt ein etwa $10 \mathrm{~cm}$ mächtiger humoser, toniger Klei, dessen Schichtung durch künstliche Einwirkung gestört ist. Wahrscheinlich ist er gepflügt. Den Abschluß der anstehenden Schichten unter der Warft bildet ein $10 \mathrm{~cm}$ mächtiger, humoser, feingeschichteter Klei. Die Basis der Warft liegt zwischen $\mathrm{NN}+0,2$ und NN $+0,8 \mathrm{~m}$. Die Warft selbst läßt mehrere Bauphasen erkennen, die älteste besteht aus einem Kern aus Klei und Torfstücken, die seitlich durch aufgeschichtete Kleisoden gestützt und auch oben durch Kleisoden abgedeckt werden. Auf dieser Kernwarft haben die ersten Siedler im 13. Jahrhundert gewohnt. Später wurde die Warft nur noch mit Klei erhöht und vergrößert. Es fällt auf, daß die unter der Warft erhaltene Marschoberfläche höher liegt als die heutige um die Warft herum. Demnach muß hier der höchste Teil des Bodens, der, wie unter der Warft sichtbar, nur aus einer dünnen Kleidecke und darunter Torf bestand, abgetragen worden sein, wie übrigens auf großen Teilen der Insel Pellworm.

Die Untersuchung des Profils am Punkt 14, gleichfalls einer Warft, ergab ganz andere Befunde (Abb. 2). Ahnlich wie in der gerade beschriebenen Siedlungsstelle 5 war als tiefste Schicht ein toniger, stark schilfhaltiger Klei im Profil aufgeschlossen, dessen Oberkante im Zentrum der Warft bei NN $-1,35 \mathrm{~m}$ und an den Seiten bis maximal NN -0,2 $\mathrm{m}$ hoch liegt. Darüber folgt eine etwa $20 \mathrm{~cm}$ mächtige Schilftorfschicht, die zur westlichen Seite der Warft hin allmählich ausdünnt. Ihr Alter ist $2330 \pm 55$ Jahre BP (Hv-7394). Darüber folgt ein bis zu 0,45 m mächtiger humoser, schilfhaltiger, toniger Klei, dessen Humusgehalt nach oben hin abnimmt. Unmittelbar unter der Oberkante ist eine bis zu $2 \mathrm{~cm}$ mächtige Feinsandschicht, offenbar die Ablagerung einer einzelnen Sturmflut, eingeschaltet. Die Oberkante dieses ersten Schichtpaketes über dem Torf bildet ein bis zu $5 \mathrm{~cm}$ mächtiger Humusdwog, der scharf gegen das Hangende abgegrenzt ist. Er liegt zwischen $\mathrm{NN}-0,3 \mathrm{~m}$ an der Seite der Warft und $\mathrm{NN}-1,0 \mathrm{~m}$ unter dem Zentrum der Warft. Das zweite Schichtpaket besteht aus $50 \mathrm{~cm}$ mächtigen, schwach humosen, tonigen Schichten, die im unteren Teil mit feinen Sandlagen durchsetzt sind. Darüber hat sich ebenfalls ein Humusdwog gebildet. Bemerkenswert ist, daß sich in dieses Schichtpaket mehrere Rinnen eingetieft haben, die später wieder mit schilfhaltigem Klei zuschlickten. Da die Gefahr einer Durchwurzelung von Schilf aus jüngerem Klei an dieser Stelle nicht gegeben war, wurde hier eine ${ }^{14} \mathrm{C}$-Analyse des Schilfes durchgeführt, die ein Alter von $1620 \pm 60$ Jahre BP (Hv-7395) ergab. Die Oberfläche über diesem Schichtpaket bildete sich erst, nachdem die Gräben bereits zugeschlickt waren. Das dritte Schichtpaket besteht aus geschichteten, überwiegend schluffigen Sedimenten von $40-70 \mathrm{~cm}$ Mächtigkeit und wird durch einen humosen braunen, $1-2 \mathrm{~cm}$ mächtigen Bodenhorizont abgeschlossen, auf den die Warft aufgesetzt worden ist, die nur aus Klei besteht und nach der gefundenen Keramik erstmals im 14. Jahrhundert besiedelt war.

\section{Ergebnisse}

In den Profilen, die nur über eine kleine Fläche verteilt sind, deutet der Torf (Punkt 5 und 14) und der Boden unter der Kulturschicht (Punkt 3) erstmals eine Unterbrechung der 
klastischen Sedimentation an, in deren Verlauf weit über $10 \mathrm{~m}$ Sedimente über der Holozänbasis im Bereich Pellworms abgelagert wurden. Wahrscheinlich wurde dies durch eine Barre verursacht, die westlich von Pellworm lag und von einem dort vorhandenen Geestkern, vielleicht dem von Ditrmer (1952) nachgewiesenen Hever-Geestkern, ernährt wurde. Daß diese vermutete Barre nicht unmittelbar vor Pellworm gelegen hat, ist schon daraus zu ersehen, daß der Torf auch noch westlich von Pellworm und sogar westlich von Hooge im Watt zu finden ist. Nach den dargelegten Befunden gab bzw. gibt es noch heute Gebiete auf Pellworm, in denen der Torf von mächtigen Ablagerungen überdeckt ist, solche mit nur einer geringen Kleibedeckung über dem Torf, wo allerdings heute der Torf im Zuge der Verfehnung weitgehend abgegraben ist; ferner gab es einige Gebiete, die so hoch lagen, daß sich kein Torf bilden konnte, hier entstand ein Boden. Es ist unwahrscheinlich, daß die Oberfläche, über die sich der Torf ausgebreitet hat, bei Beginn der Torfbildung solche Höhenunterschiede aufwies, wie wir sie heute feststellen, sie können höchstens wenige Dezimeter betragen haben. Um aus den geschilderten Befunden Wasserstandshöhen ableiten zu können, muß Sackung und Setzung des Untergrundes abgeschätzt werden. Dies kann jedoch nur in grober Annäherung geschehen. Vergleicht man die heutige Höhenlage der Torfoberkante unter der Warf 5 mit der des Bodens unter der Siedlung 3, so fällt auf, daß der Torf teilweise genau so hoch liegt wie der Boden. Dies kann jedoch zur Zeit der Torfentstehung nicht so gewesen sein; damals muß die Bodenoberfläche höher gelegen haben. Der Torf unter der Siedlung 5 erreicht heute an der höchsten Stelle eine Höhe von $\mathrm{NN}+0,6 \mathrm{~m}$ und hat hier eine Mächtigkeit von $28 \mathrm{~cm}$. Unterstellt man für die Sackung und Setzung einen Betrag von einem Drittel der ursprünglichen Mächtigkeit, ergibt sich als frühere Höhenlage für die Torfoberfläche ca. NN $+0,75 \mathrm{~m}$. Dies müßte ungefähr die Höhe des Grundwasserspiegels am Ende der Torfbildung gewesen sein. Für eine wesentliche Sackung und Setzung des Untergrundes an dieser Stelle gibt es keine Hinweise. Die tonigen Schichten des Untergrundes haben nur geringe Mächtigkeit und das Gewicht der Warft hat nur im Bereich großer Torfmächtigkeiten Setzungen verursacht (Abb. 2).

Der Boden der Flachsiedlung erreicht heute noch eine Höhe von NN + 0,60 m. Demnach wäre hier eine Sackung und Setzung des Untergrundes von mindestens 0,15 m anzunehmen, um die ursprüngliche Höhe der Torfoberkante zu erreichen. Da die obersten Schichten unter dem Boden jedoch Sturmflutschichten sind, muß man davon ausgehen, daß der Boden ursprünglich noch höher gelegen haben muß als die Torfoberkante, die ja etwa die Höhe des Grundwasserspiegels anzeigt, dessen Höhe in diesem Gebiet wohl nahe der des MThw gelegen haben dürfte, da durch die im Westen vermutete Barre die Vorflutverhältnisse zweifellos ungünstig waren und sich das Wasser im Hinterland gestaut hat. Die Sturmflutschichten unter Siedlung 3 sind noch über diesem Niveau entstanden, demnach muß für die letzte Hälfte des ersten vorchristlichen Jahrtausends, also die Zeit vor der Torfbildung, für das Mittlere Tidehochwasser eine Höhe angenommen werden, die bei $\mathrm{NN}+$ 0,8 m gelegen hat. Bei der von Linke (1979) angegebenen MThw.-Höhe für Pellworm ist die Sackung und Setzung nicht berücksichtigt.

Die ungewöhnlich tiefe Lage des Torfes am Punkt 14 mit bis zu NN -1,2 m ist einerseits auf die Setzung des Untergrundes durch die Auflast der Warft verursacht $(0,6 \mathrm{~m})$ und andererseits durch die Sackung der liegenden Schichten des Torfes nach dessen Entstehung. Uber dem Torf liegen 3 durch Humusdwöge abgegrenzte Sedimentdecken, die zusammen bis zu $1,7 \mathrm{~m}$ mächtig sind. Ahnlich mächtige Schichten sind unter den anderen Siedlungen nicht vorhanden. Demnach kann man davon ausgehen, daß sich nach dem Ende der Torfbildung nur dort noch Sedimente ablagern konnten, wo die Oberfläche besonders niedrig war. Für die erste Hälfte des ersten nachchristlichen Jahrtausends muß daher angenommen werden, daß der Meeresspiegel nicht so hoch lag, wie zur Zeit der Torfentste- 
hung. Nach einem besonders hohen Stand des MThw muß für die Zeit nach Chr. Geb. zunächst ein Abfallen angenommen werden. Eine Bestätigung für diese Annahme ist auch, daß bisher aus Nordfriesland nirgends Ablagerungen aus dem frühen ersten nachchristlichen Jahrtausend nachgewiesen werden konnten, die über der hohen alten Marsch der römischen Kaiserzeit liegen. Erst im 14. Jahrhundert wurden diese Gebiete wieder von Sedimenten bedeckt (PRANGe 1963, 1967).

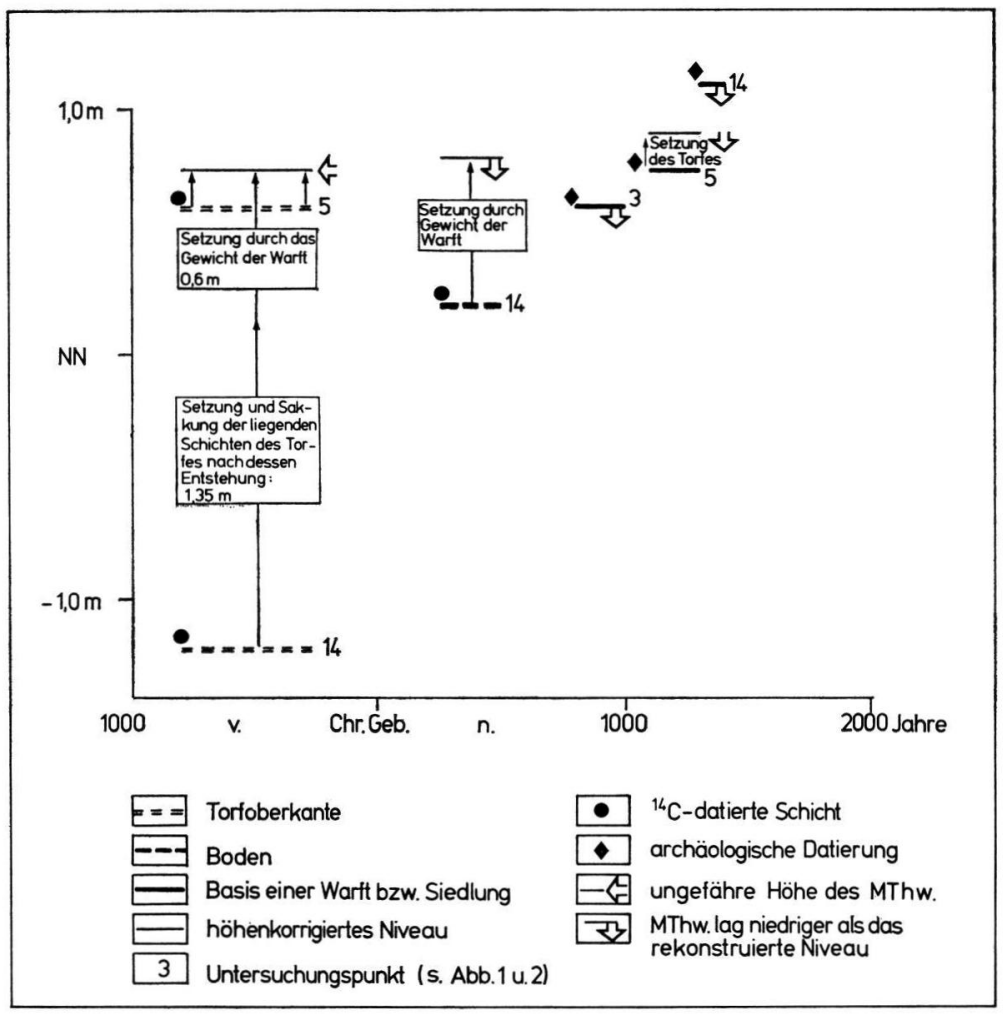

Abb. 3: Sackungs- und setzungskorrigiertes Zeit-Höhen-Diagramm für die datierten Schichten der beschriebenen Profile. Die ${ }^{14} \mathrm{C}$-Daten wurden dendrochronologisch korrigiert nach WILLKomm (1976).

Für die Punkte 3 und 5 ist weiterhin der Zeitraum zu betrachten, an dem die Siedlungen angelegt wurden. Demnach konnte man im 9./10. Jahrhundert noch auf hohen Stellen der flachen Marsch siedeln, ohne allzu häufig von Sturmfluten gestört zu werden. Offenbar hatte der Wasserstand noch nicht wieder die Höhe erreicht, die er vor der Bodenbildung an dieser Stelle hatte. Im 13. Jahrhundert war diese Siedlungsweise scheinbar nicht mehr möglich, zu dieser Zeit wurden erste Warften gebaut, z. B. am Punkt 5, das bedeutet, daß spätestens seit dem Ende des ersten nachchristlichen Jahrtausends die Sturmflutwasserstände wieder anstiegen und damit wohl auch das MThw. 


\section{Schriftenverzeichnis}

Bantelmann, A. (1966): Die Landschaftsentwicklung an der schleswig-holsteinischen Westküste. Die Küste, 14, 2: 5-99, 51 Abb.; Heide/Holst.

DitTmer, E. (1952): Die nacheiszeitliche Entwicklung der schleswig-holsteinischen Westküste. Meyniana, 1: 138-168, 7 Abb.; Neumünster.

Higelke, B., Hoffmann, D. \& Müller-Wille, M. (1976): Zur Landschaftsentwicklung und Siedlungsgeschichte der nordfriesischen Marscheninseln und Watten im Einzugsbereich der Norderhever. - Probleme der Küstenforschung im südlichen Nordseegebiet, 11: 163-185, 13 Abb.; Hildesheim.

Higelke, B., Hoffmann, D., KüHn, H. J. \& Müller-Wille, M. (1979): Geowissenschaftlich-archäologische Untersuchungen zur Landschafts- und Siedlungsgeschichte von Nordfriesland. Archäologisches Korrespondenzblatt, 9: 223-239, 9 Abb., 3 Taf.; Mainz.

LiNkE, G. (1979): Ergebnisse geologischer Untersuchungen im Küstenbereich südlich Cuxhaven. Probleme der Küstenforschung im südlichen Nordseegebiet, 13: 39-83, 18 Abb., 1 Tab.; Hildesheim.

Prange, W. (1963): Das Holozän und seine Datierung in den Marschen des Arlau-Gebietes, Nordfriesland. - Meyniana, 13: 47-76, $11 \mathrm{Abb}$; Kiel.

- (1965): Die Höhe der Sturmflut vom 11. Oktober 1634 in Nordfriesland nach neuen Wasserstandsmarken. - Z wischen Eider und Wiedau, Heimatkalender für Nordfriesland, 1965: 40-48, $1 \mathrm{Abb} . ;$ Husum.

- (1967): Geologie des Holozäns in den Marschen des nordfriesischen Festlandes. - Meyniana, 17: 45-94, 20 Abb., 2 Taf., 1 Tab.; Kiel.

WiLLкомм, H. (1976): Altersbestimmungen im Quartär. - 276 S.; München.

Manuskript eingegangen am 3. 1. 1980. 